\title{
Points of low height on elliptic surfaces with torsion
}

\author{
Sonal Jain
}

\begin{abstract}
We determine the smallest possible canonical height $\hat{h}(P)$ for a non-torsion point $P$ of an elliptic curve $E$ over a function field $\mathbb{C}(t)$ of discriminant degree $12 n$ with a 2 -torsion point for $n=1,2,3$, and with a 3 -torsion point for $n=1,2$. For each $m=2,3$, we parametrize the set of triples $(E, P, T)$ of an elliptic curve $E / \mathbb{Q}$ with a rational point $P$ and $m$-torsion point $T$ that satisfy certain integrality conditions by an open subset of $\mathbb{P}^{2}$. We recover explicit equations for all elliptic surfaces $(E, P, T)$ attaining each minimum by locating them as curves in our projective models. We also prove that for $n=1,2$, these heights are minimal for elliptic curves over a function field of any genus. In each case, the optimal $(E, P, T)$ are characterized by their patterns of integral points.
\end{abstract}

\section{Introduction}

Let $E$ be an elliptic curve over a field $K$ of characteristic 0 . Néron and Tate showed, independently, that there is a canonical height function

$$
\hat{h}: E(K) \longrightarrow[0, \infty)
$$

that descends to a positive definite quadratic form on $E(K) / E(K)_{\text {tors }}$. If $K$ is a function field, then $\hat{h}$ takes its values in $\mathbb{Q}$. Let $P$ be a non-torsion point in $E(K)$. It is natural to ask how small $\hat{h}(P)$ can be. In the case where $K$ is a number field, if one bounds the minimal discriminant of $E$ then there are only finitely many isomorphism classes of curves, and it is a finite problem to find the smallest non-zero canonical height.

In the case that $K=k(C)$ is a function field of characteristic zero, one may fix the discriminant degree of the elliptic curve $E / K$, that is, the degree of the discriminant considered as a divisor on $C$. For $K=\mathbb{C}(t)$ this is the degree of the minimal discriminant polynomial $\Delta(t)$. The discriminant degree is always a multiple of 12 , and an elliptic curve $E / k(C)$ of discrimant degree $12 n$ is equivalent to an elliptic surface $\mathcal{E} / k$ fibred over $C$ of arithmetic genus $n$.

If $d>12$ then there are infinitely many elliptic curves over $K$ of discriminant degree $d$ (see [12, Appendix 1]). For fixed $d$, however, the set of values of $\hat{h}$ is discrete. One may ask, for fixed $d$, what is the smallest possible canonical height $\hat{h}(P)$ of a non-torsion point?

Following the notation in [3], we let $\hat{h}_{\min }(g, 12 n)$ denote the minimal height of a non-torsion point on an elliptic curve over a function field of genus $g$ of discriminant degree $d=12 n$, and let $\hat{h}_{\text {min }}(12 n)$ denote the minimal height of a non-torsion point on an elliptic curve of discriminant degree $12 n$.

In [12], Oguiso and Shioda classified all rational elliptic surfaces, and as a result determined $\hat{h}_{\min }(0,12)=1 / 30$. Nishiyama, in $[\mathbf{1 1}]$, determined $\hat{h}_{\min }(0,24)=11 / 420$, but was unable to write down any examples of elliptic surfaces attaining this minimum. Elkies, in [3], used different techniques to determine that $\hat{h}_{\min }(12)=\hat{h}_{\min }(0,12)=1 / 30, \hat{h}_{\min }(24)=\hat{h}_{\min }(0,24)=$ $11 / 420$ and $\hat{h}_{\min }(0,36)=23 / 840$. In addition he exhibited the general equations for all pairs $(E, P)$ attaining the minima, characterizing the optimal surfaces for each $d$ as those having the greatest possible numbers of integral multiples of a point $P$.

Received 18 May 2009; revised 26 March 2010.

2000 Mathematics Subject Classification 11G50.

This work was partially supported by the NSF RTG grant DMS-0739380. 
As most elliptic surfaces have trivial torsion, one expects that the smallest positive height among those $E$ with a non-trivial torsion subgroup should be greater than that for general $E$. In this paper we use an approach similar to that of Elkies in [3] to compute minimum heights for elliptic surfaces with small $d$ in the cases where $E(K)_{\text {tors }}=\mathbb{Z} / 2 \mathbb{Z}$ and $\mathbb{Z} / 3 \mathbb{Z}$. We also characterize the elliptic surfaces attaining the minima by their patterns of integral points. As one might expect, the surfaces tend to have many integral points. We build projective models in which we are able to recover explicit equations for all curves attaining the minima. All of the elliptic surfaces inherit symmetries from the moduli spaces in which they are recovered. For $E(K)_{\text {tors }}=\mathbb{Z} / 2 \mathbb{Z}$, we show that $\hat{h}_{\min }(12, \mathbb{Z} / 2 \mathbb{Z})=\hat{h}_{\min }(0,12, \mathbb{Z} / 2 \mathbb{Z})=1 / 12, \hat{h}_{\min }(24, \mathbb{Z} / 2 \mathbb{Z})=$ $\hat{h}_{\min }(0,24, \mathbb{Z} / 2 \mathbb{Z})=3 / 40$, and $\hat{h}_{\min }(0,36, \mathbb{Z} / 2 \mathbb{Z})=3 / 40$. For $E(K)_{\text {tors }}=\mathbb{Z} / 3 \mathbb{Z}$, we show that $\hat{h}_{\min }(12, \mathbb{Z} / 3 \mathbb{Z})=\hat{h}_{\min }(0,12, \mathbb{Z} / 3 \mathbb{Z})=1 / 6, \hat{h}_{\min }(24, \mathbb{Z} / 3 \mathbb{Z})=\hat{h}_{\min }(0,24, \mathbb{Z} / 3 \mathbb{Z})=1 / 6$. A precise statement of our results is given in $\S 2$.

\subsection{Further problems}

It is interesting to ask what is the global minimum for the canonical height $\hat{h}(P)$ of a non-torsion point $P$ of an elliptic curve over a function field. Elkies has found elliptic curves $(E, P)$ over $\mathbb{C}(t)$ with $\hat{h}(P)=41 / 1540$ and $\hat{h}(P)=261 / 10010[3, \S 1.3]$. The $41 / 1540$ curve is conjectured to be the minimum for $d=48$, and the $261 / 10010$ is conjectured to be the minimum for $d=60$. We have found an elliptic curve $E / \mathbb{C}(t)$ of discriminant degree 84 with a non-torsion $P$ of canonical height $\hat{h}(P)=2987 / 120120$ [6]. This is currently the smallest known, and we have heuristics suggesting that this may be a global minimum.

One may also ask asymptotic questions about the behavior of $\hat{h}_{\min }(g, d)$ for fixed $g$ as $d \rightarrow \infty$. Hindry and Silverman proved that there exists a constant $C>0$ such that

$$
\hat{h}_{\min }(g, 12 n) \geqslant C n-O_{g}(1)
$$

proving effectively a conjecture of Lang [9] in the function field case. The error terms $O_{g}(1)$ vanish for $g=0,1$. They computed an explicit $C \approx 7 \cdot 10^{-10}$ [5]. Elkies improved the value of $C \approx 5 \cdot 10^{-4}$, and conjectured what the best possible value for $C$ should be [2]. In a future paper we compute explicit values for the constant $C$ for elliptic curves with a 2-or 3-torsion point, and compare these values with the Birch and Swinnerton-Dyer formula. We also examine the asymptotic behavior of the minimum regulator $R_{\min }^{2}(g, d)$ of a rank two subgroup of $E$ as $d \rightarrow \infty$. More generally, we describe the region in the three-dimensional space of reduced binary forms that is asymptotically obtainable by Mordell-Weil lattices in rank two, proving that the boundary of the region is cut out by algebraic equations.

One should be able to prove results analogous to Theorems 1-5 (see $\S 2$ ) for other small torsion groups of elliptic surfaces. One can also examine similar questions for higher-rank subgroups. In another paper [7], we compute the minimal regulators for rank two sublattices of rational and $K 3$ elliptic surfaces, again finding explicit equations for all surfaces attaining the minima. We use similar ideas to build a $\mathbb{P}^{3}$ model in which to recover the optimal surfaces.

\section{Results}

Let $\hat{h}_{\min }(g, 12 n, G)$ denote the minimal height of a non-torsion point on an elliptic curve $E$ over a function field $K$ of genus $g$ with torsion subgroup $E(K)_{\text {tors }}=G$. Let $\hat{h}(12 n, G)$ denote the minimum for an elliptic curve over a function field of any genus. Our results are listed below.

THEOREM 1.

(i) (Oguiso and Shioda [12]) $\hat{h}_{\min }(0,12, \mathbb{Z} / 2)=1 / 12$.

(ii) $\hat{h}_{\min }(12, \mathbb{Z} / 2 \mathbb{Z})=1 / 12$. Moreover, let $E$ be an elliptic curve of discriminant degree $d=12$ with a 2 -torsion point $T_{2}$ over a complex function field $K$, and let $P \in E(K)$. Then the following are equivalent: 
(a) $\hat{h}(P)=1 / 12$;

(b) each of $P, 2 P, 3 P, 4 P$ is an integral point on $E$;

(c) $K \cong \mathbb{C}(t)$, and $\left(E, P, T_{2}\right)$ is equivalent to the curve

$$
E(q): y^{2}=x^{3}+\left(t^{2}-2 q t-2\right) x^{2}-\left(t^{2}-1\right)(q t+1)^{2} x
$$

over the t-line with the rational point

$$
P:(x, y)=((t+1)(q t+1), t(q t+1)(t+1))
$$

and 2-torsion point $T_{2}=(0,0)$, for some $q \in \mathbb{C}$ other than 0 or \pm 1 .

The symmetry $t \leftrightarrow-t$ interchanges $P$ and $P+T_{2}$ on $E(q)$, and is inherited from the moduli space in which we recover the family (see $\S 5.1$ ). Specializing the above family to $q=0$ forces $4 P=0$ but $2 P \neq T_{2}$, and thus $E(0)(\mathbb{C}(t))_{\text {tors }}=\mathbb{Z} / 4 \mathbb{Z} \oplus \mathbb{Z} / 2 \mathbb{Z}$. This gives us equations for the universal elliptic curve with torsion subgroup $\mathbb{Z} / 4 \mathbb{Z} \oplus \mathbb{Z} / 2 \mathbb{Z}$. Specializing to $q= \pm 1$ forces $3 P=T_{2}$, which gives $E( \pm 1)(\mathbb{C}(t))_{\text {tors }}=\mathbb{Z} / 6 \mathbb{Z}$. This gives equations for $X_{1}(6)$, the universal elliptic curve with a 6 -torsion point.

\section{THEOREM 2.}

(i) $\hat{h}_{\min }(0,24, \mathbb{Z} / 2)=3 / 40$.

(ii) $\hat{h}_{\min }(24, \mathbb{Z} / 2 \mathbb{Z})=3 / 40$. Moreover, let $E$ be an elliptic curve of discriminant degree $d=24$ with a 2 -torsion point $T_{2}$ over a complex function field $K$, and let $P \in E(K)$. Then the following are equivalent:

(a) $\hat{h}(P)=3 / 40$;

(b) each of $P, \ldots, 5 P$ is an integral point on $E$;

(c) $K \cong \mathbb{C}(t)$, and $\left(E, P, T_{2}\right)$ is equivalent to the curve

$$
\begin{aligned}
E: y^{2}= & x^{3}+\left((t-1)^{2}(1+3 t)^{2}-8 t(t+1)^{2}\right) x^{2} \\
& +16 t^{2}\left((t+1)^{4}-(t-1)^{2}(1+3 t)^{2}\right) x
\end{aligned}
$$

over the $t$-line with the rational point

$$
P:(x, y)=\left(16 t^{3}, 16 t^{3}(t-1)(3 t+1)\right)
$$

and 2-torsion point $T_{2}=(0,0)$.

The $K 3$ elliptic surface above also has a symmetry inherited from the moduli space in it is recovered (see $\S 5.1$ ), which interchanges $P$ and $P+T_{2}$.

THEOREM 3.

(i) $\hat{h}_{\min }(0,36, \mathbb{Z} / 2 \mathbb{Z})=3 / 40$.

(ii) Let $E$ be an elliptic curve over $\mathbb{C}(t)$ with discriminant degree $d=36$ and $P$ a rational point on $E$. Then the following are equivalent:

(a) $\hat{h}(P)=3 / 40$;

(b) each of $P, \ldots, 6 P$ is an integral point on $E$;

(c) $\left(E, P, T_{2}\right)$ is equivalent to the curve

$$
\begin{aligned}
E(q): y^{2}= & x^{3}+\left((q(2+t)((1-q) t-2 q)((4 q+2)+(2 q-1) t))^{2}\right. \\
& -2\left(t^{2}-q(2+t)((1-q) t-2 q)((4 q+2)+(2 q-1) t)\right) \\
& \cdot t(2 q+1+q t)((1-2 q) t-4 q)) x^{2} \\
& \cdot t^{4}\left(4 q+8 q^{2}-t-2 t q+8 t q^{2}-2 q t^{2}+2 q^{2} t^{2}\right) \\
& \cdot(1+2 q+t q)^{2}(2 t q+4 q-t)^{3} x
\end{aligned}
$$


over the $t$-line with the rational point $P=(x, y)$, where

$$
\begin{aligned}
x= & -t^{3}(1+2 q+q t)(2 t q+4 q-t) \\
y= & q t^{3}(2+t)(t q-t+2 q)(2 q t+4 q-t) \\
& \cdot(-t+2 t q+2+4 q(1+2 q+q t))
\end{aligned}
$$

and $T_{2}=(0,0)$, for any $q$ other than 0 or $-1 / 2$.

As in Theorems 1 and 2, $E(q)$ has a symmetry that interchanges $P$ and $P+T_{2}$. Specializing to $q=0$, we obtain a singular curve. Specializing to $q=-1 / 2$, and changing the Weierstrass equation by $a_{i} \mapsto a_{i} / t^{i}$ to get the minimal model, one obtains a $K 3$ surface with three $I_{6}$ fibers at $t=\infty,-1$ and -2 , and three $I_{2}$ fibers at $t=1,-2 / 3$ and $-4 / 3$, as well as $6 P=0$ and $3 P \neq T$. This gives us equations for the universal elliptic curve with torsion subgroup $\mathbb{Z} / 2 \mathbb{Z} \oplus \mathbb{Z} / 6 \mathbb{Z}$, an example of a singular $K 3$ surface with all of its Néron-Severi group defined over $\mathbb{Q}$. We do not claim that $\hat{h}_{\min }(36, \mathbb{Z} / 2 \mathbb{Z})=\hat{h}_{\min }(0,36, \mathbb{Z} / 2 \mathbb{Z})($ see $\S 5.3)$.

We prove the following analogous results in the case when $E$ has a 3 -torsion point $T_{3}$.

THEOREM 4.

(i) (Oguiso and Shioda $[\mathbf{1 2}]) \hat{h}_{\min }(0,12, \mathbb{Z} / 3 \mathbb{Z})=1 / 6$.

(ii) $\hat{h}_{\min }(12, \mathbb{Z} / 3 \mathbb{Z})=1 / 6$. Moreover, let $E$ be an elliptic curve of discriminant degree $d=12$ with a 3 -torsion point $T_{3}$ over a complex function field $K$, and let $P \in E(K)$. Then the following are equivalent:

(a) $\hat{h}(P)=1 / 6$;

(b) each of $P, 2 P, 3 P$ is an integral point on $E$;

(c) $K \cong \mathbb{C}(t)$, and $\left(E, P, T_{3}\right)$ is equivalent to the curve

$$
y^{2}+((1+q) t+(2-q)) x y+\left(q t^{2}+(1-q) t\right) y=x^{3}
$$

over the $t$-line with the rational point $P:(x, y)=\left(-t, t^{2}\right)$ and 3 -torsion point $T_{3}:(x, y)=(0,0)$, for some $q \in \mathbb{C}$ other than 0 or 1 .

The surfaces above have a symmetry of order three inherited from the moduli space in which they are recovered (see $\S 5.2$ ). The symmetry permutes $\{P, P+T, P+2 T\}$ cyclically by the odd permutations. Specializing to $q=1$ forces $2 P=T_{3}$ and we get equations for $X_{1}(6)$. Specializing to $q=0$ forces $2 P=-T_{3}$ and we again get equations for $X_{1}(6)$.

\section{THEOREM 5 .}

(i) $\hat{h}_{\min }(0,24, \mathbb{Z} / 3 \mathbb{Z})=1 / 6$.

(ii) $\hat{h}_{\min }(24, \mathbb{Z} / 3 \mathbb{Z})=1 / 6$. Moreover, let $E$ be an elliptic curve of discriminant degree $d=24$ with a 3 -torsion point $T$ over a complex function field $K$, and let $P \in E(K)$. Then the following are equivalent:

(a) $\hat{h}(P)=1 / 6$

(b) each of $P, 2 P, P \pm T, 2 P \pm T, 3 P \pm T$ is an integral point on $E$;

(c) $K \cong \mathbb{C}(t)$, and $(E, P, T)$ is equivalent to the curve

$$
y^{2}+q\left(q^{2}-t\right)^{2} x y+q^{2} t^{2}(q-t)^{2} y=x^{3}
$$

over the $t$-line with the rational point

$$
P=(x, y)=\left(-q^{2} t(q-t)^{2}, t^{2}(q-t)^{3}\right)
$$

and 3-torsion point $T_{3}=(x, y)=(0,0)$, where $q$ satisfies $q^{2}-q+1=0$.

The surfaces above also inherit the symmetry of order three which permutes $\{P, P+T, P+$ $2 T\}$ cyclically by the odd permutations. We do not state a result for $n=3$ in the case where $E(K)_{\text {tors }}=\mathbb{Z} / 3 \mathbb{Z}$. We found that $\hat{h}_{\min }(0,36, \mathbb{Z} / 3 \mathbb{Z})=1 / 6$ in this case as well, though we found 
multiple configurations that achieve this height. There was also no nice characterization of these configurations in terms of their patterns of integral points.

\subsection{Outline of methods}

In $[7, \S 3]$, we define a map

$$
\{(E, P, Q)\} \longrightarrow \mathcal{G}=\mathbb{Z}\left[\mathcal{A} / D_{\infty}\right],
$$

which associates to each elliptic surface $E$ and pair of sections $P, Q \in E(K)$ an element $\gamma$ in a free abelian group $\mathcal{G}$ (see $\S 3.3$ ). From this element $\gamma$ one can compute the correction terms for the local heights of $m P+m^{\prime} Q$, a lower bound for the conductor degree of $E$, and the discriminant degree of $E$. We fix the discriminant degree $12 n$ and search through the finite number of elements of $\mathcal{G}$ that could correspond to triples $(E, P, T)$, with $P$ a non-torsion point of small canonical height, and $T$ a 2 - or 3-torsion point. We then compute lower bounds for the canonical height $\hat{h}(P)$.

In each case, the lower bound is attained. We use the fact that the optimal configurations must correspond to elliptic surfaces that have several integral points to find equations. We parametrize the set of triples $(E, P, T)$, where $E$ is an elliptic curve over $\mathbb{Q}, P \in E(\mathbb{Q})$ a non-torsion point, and $T \in E(\mathbb{Q})$ a 2-torsion point, with the property that $P, 2 P, P+T$ and $2 P+T$ are all integral by an open subset of $\mathbb{P}^{2}$. A curve $C$ of degree $n$ in this $\mathbb{P}^{2}$ is equivalent to an elliptic surface fibred over $C$ of discriminant degree $12 n$, together with a section $P$ and a 2-torsion section $T$ such that $P, 2 P, P+T$ and $2 P+T$ are all integral (that is, they do not meet the zero section). We recover the $d=12,24,36$ surfaces as rational curves of degree $n=1,2,3$ in this moduli space. Similarly, we parametrize the set of triples $(E, P, T)$ of $E / \mathbb{Q}$ with a non-torsion point $P$ and a 3 -torsion point $T$, and the property that $P, P+T$ and $P-T$ are all integral by an open subset of $\mathbb{P}^{2}$, and recover the elliptic surfaces in Theorems 4 and 5 as curves in this moduli space.

Our parametrizations make use of Tate's trick [14], which Tate used to parametrize the moduli space $X_{1}(N)$ of elliptic curves with an $N$-torsion point for small $N$. Tate's trick was first applied in the way we use it by Elkies [4], who parametrized the set of pairs of an elliptic curve with a rational point $(E, P)$ such that $P, \ldots, 4 P$ are all integral.

We summarize the basic strategy of the paper as follows.

(1) Find the element $\gamma \in \mathcal{G}$ that yields a lower bound for $\hat{h}(P)$.

(2) Show that $\gamma$ is the unique configuration that could yield an elliptic surface with the pattern of integral points listed in part (ii)(b).

(3) Construct the general $(E, P, T)$ with configuration equal to $\gamma$ by recovering it as a curve in the corresponding moduli space of each theorem.

To show the result over higher-genus curves, we take advantage of the moduli spaces we construct in $\S 5$. We show that each configuration that could possibly yield an $(E, P, T)$ over a higher-genus curve with very small $\hat{h}(P)$ has a pattern of integral points that would imply it could be recovered as a curve of degree $n$ in the corresponding $\mathbb{P}^{2}$ model. As curves of degree $n=1,2$ in $\mathbb{P}^{2}$ are rational, this will imply the result over higher-genus curves. We deal with these configurations in $\S 5.3$.

\section{Setup}

In this section we discuss the essential tools we will need to use in our proofs of Theorems 1-5. We begin by defining the naive height. Whenever possible, we give references instead of proofs.

\subsection{Naive heights and local correction terms}

To a non-constant elliptic curve $E / k(C)$, one can associate a smooth projective surface $\mathcal{E} / k$ with a relatively minimal fibration $\pi: \mathcal{E} \longrightarrow C$ such that the generic fiber is an elliptic curve, 
no fibers contain exceptional curves of the first kind, and the surface is non-constant. This surface is called the Kodaira-Néron model of $E$ (see $[\mathbf{8}, \mathbf{1 0}]$ for a background). The group $E(k(C))$ is isomorphic to the group of sections of $\pi$ defined over $\mathbb{C}$. For a background on elliptic surfaces, see Chapter 3 of Silverman's book [13].

We define the naive height $h(P)$ of a point $P \in E(k(C))$ to be twice the intersection number of the section $s_{P}$ corresponding to $P$ and the zero section $s_{0}$. For more on this definition, see $\S 2.3$ of our paper [7]. The standard definition of the naive height is $2 s_{P} \cdot s_{0}+2 n$, and hence differs from our definition by $2 n$. We use this somewhat unconventional definition because points of naive height zero will be precisely those points that are integral.

The canonical height $\hat{h}(P)$ can be described as the sum of the naive height $h(P)$ and local correction terms:

$$
\hat{h}(P)=h(P)+\sum_{v} \lambda_{v}(P) .
$$

The correction terms can be computed from the Kodaira-Néron model of $E$, and are given below. The formulas were worked out by Cox and Zucker in [1]. For explicit formulas consistent with our definitions and notations, see $[7, \S 2.6]$. We will note one particular formula here, as it is important for our definitions in the following section.

If $E_{v}$ is a multiplicative fiber of type $I_{\nu}$ and $s_{P}$ passes through component $a$, then

$$
\lambda_{v}(P)=\nu B(a / \nu),
$$

where $B(x)=\langle x\rangle^{2}-\langle x\rangle+1 / 6$ is the second Bernoulli function of $x$. The quantity $\langle x\rangle$ denotes the fractional part of $x$.

We also observe that in the formulas in $[7, \S 2.6]$ that the correction terms are bounded above and below:

$$
-d_{v} / 12 \leqslant \lambda_{v}(P) \leqslant d_{v} / 6
$$

Here $d_{v}$ is the local discriminant degree at $v$. Summing over $v$, if $d=12 n$ is the discriminant degree we obtain the following bound on the difference between the naive and canonical heights:

$$
-n \leqslant \hat{h}(P)-h(P) \leqslant 2 n .
$$

We use this observation extensively in our search in $\S 4$.

\subsection{Conditions used to eliminate configurations}

Below, we list the facts we must use about canonical heights on elliptic surfaces. Each of these facts is discussed in detail in our paper [7] as well as Elkies' paper [3]. These conditions will be used both in our definition of the group $\mathcal{G}$, as well as our search through elements of $\mathcal{G}$ for configurations that might correspond to elliptic surfaces.

(1) The naive height $h(P)$ takes values in $\{0,2,4,6, \ldots\}$ and satisfies $h\left(m^{\prime} P\right) \leqslant h(m P)$ for $m^{\prime} \mid m$ and $m P \neq 0$.

(2) The canonical height can be written as a sum of local terms. If $m P \neq 0$ then

$$
\hat{h}(m P)=h(m P)+\sum_{v} \lambda_{v}(m P)
$$

where the sum is taken over places $v \in \mathbb{C}(C)$ at which the fiber $E_{v}$ is reducible.

(3) The local correction term $\lambda_{v}(m P)$ depends only on the Kodaira type of fiber $E_{v}$ and component $c_{v}$ of $E_{v}$ meeting $P$.

(4) Shioda's inequality: if $E$ has a non-torsion point then the conductor degree $N$ of $E$ is at least

$$
N \geqslant(d / 6)+\chi(C)+1
$$




\subsection{Notation}

We follow the notation given in $[\mathbf{7}, \S 3]$. Define the following equivalence relation on $\mathbb{Z}^{3}$ : set $(x, y \mid z) \sim(u, v \mid w)$ when $x w=u z$ and $y w=v z$. Each equivalence class has a unique representative $[x, y \mid z]$ with $\operatorname{gcd}(x, y, z)=1$ and $z \geqslant 0$. Let $\mathcal{A}$ denote the quotient of $\mathbb{Z}^{3}$ by this equivalence relation. The infinite dihedral group $D_{\infty}$ acts on $\mathcal{A}$ by $[x, y \mid z] \mapsto[z-x$, $z-y \mid z]$ and $[x, y \mid z] \leftrightarrow[x+z, y+z \mid z]$. Let $\mathcal{G}=\mathbb{Z}\left[\mathcal{A} / D_{\infty}\right]$ be the group of formal $\mathbb{Z}$-linear combinations of orbits of $\mathcal{A}$ under the action of $D_{\infty}$.

We associate to each triple $(E, P, Q)$ of a curve $E / K$ and points $P, Q \in E(K)$, an element $\gamma \in \mathcal{G}$ from which one can calculate discriminant degree, conductor degree, and local height corrections for $m P+m^{\prime} Q$. In the case where $E$ has semistable reduction this association is defined in the following way.

If $E_{v}$ is a fiber of $E$ of type $I_{\nu}$ lying over $v$, then the group of multiplicity one components $E_{v} /\left(E_{v}\right)_{0}$ is isomorphic to $\mathbb{Z} / \nu(v) \mathbb{Z}$. If $P$ and $Q$ intersect the fiber at the components $a(v)$ and $b(v) \in \mathbb{Z} / \nu(v) \mathbb{Z}$ respectively, we set $\gamma \in \mathcal{G}$ equal to

$$
\gamma=\sum_{v} \gamma_{v}=\sum_{v} \operatorname{gcd}(a(v), b(v), \nu(v)) \cdot[a(v), b(v) \mid \nu(v)] .
$$

Suppose that $[a, b \mid \nu] \in \mathcal{A}$ with $\nu>0$ and $\operatorname{gcd}(a, b, \nu)=1$. We define homomorphisms $\lambda_{\left(m, m^{\prime}\right)}: \mathcal{G} \longrightarrow \mathbb{Q}$ and $\mathbf{d}, \mathbf{N}: \mathcal{G} \longrightarrow \mathbb{Z}$ on the generators of $\mathcal{G}$ and extend linearly:

$$
\lambda_{\left(m, m^{\prime}\right)}([a, b \mid \nu]):=\nu B_{2}\left(\left(m a+m^{\prime} b\right) / \nu\right), \quad \mathbf{d}([a, b \mid \nu]):=\nu, \quad \mathbf{N}([a, b \mid \nu]):=1 .
$$

The height corrections $\hat{h}\left(m P+m^{\prime} Q\right)-h\left(m P+m^{\prime} Q\right)$ and the discriminant degree are the images of $\gamma$ under the homomorphisms $\lambda_{\left(m, m^{\prime}\right)}$ and $\mathbf{d}$. Each $v$ contributes 1 to the conductor $N$. Since $\operatorname{gcd}(a(v), b(v), \nu(v))=1$, the conductor degree $N \leqslant \mathbf{N}(\gamma)$. We obtain formula (2) easily from the formulas for the height correction terms $\lambda_{v}(P)$ in $\S 3.1$ :

$$
\begin{gathered}
\hat{h}\left(m P+m^{\prime} Q\right)=h\left(m P+m^{\prime} Q\right)+\lambda_{\left(m, m^{\prime}\right)}(\gamma) \quad \text { for }\left(m, m^{\prime}\right) \in \mathbb{Z}^{2} \backslash(0,0) . \\
12 n=d=\mathbf{d}(\gamma) . \\
\mathbf{N}(\gamma) \geqslant N \geqslant d / 6+(2-2 g)+r \geqslant \frac{1}{6} \mathbf{d}(\gamma)+4-2 g .
\end{gathered}
$$

For the conductor inequality, we assume the rank $r \geqslant 2$. If $g=\operatorname{gcd}(a(v), b(v), \nu(v))>1$, then we are replacing the $I_{\nu}$ fiber at $v$ with $g$ fibers of type $I_{\nu / g}$, and the values of $\lambda_{\left(m, m^{\prime}\right)}$, d, and $\mathbf{N}$ do not change. For a fiber of type $I_{0}$ we write [0].

For a curve with some additive fibers, the definition is given in [7, Table 2 of $\S 3]$. The important thing to note is the following proposition.

Proposition 6. Let $E$ be an elliptic curve over a function field $K$ of genus $g$ and $P, Q \in$ $E(K)$. For each singular fiber $E_{v}$ define an element $\gamma_{v} \in \mathcal{G}$ according to [7, Table 2]. Then:

(i) $\lambda_{v}\left(m P+m^{\prime} Q\right)=\lambda_{\left(m, m^{\prime}\right)}\left(\gamma_{v}\right)$ for each $\left(m, m^{\prime}\right) \in \mathbb{Z}^{2} \backslash(0,0)$;

(ii) $d_{v}=\mathbf{d}\left(\gamma_{v}\right)$;

(iii) $N_{v} \leqslant \mathbf{N}\left(\gamma_{v}\right)$.

Proof. The first statement can be verified by comparing the values arrived at by the table to the values arrived at by using formulas in $[\mathbf{7}, \S 2.6]$. The other two statements are immediate.

This proposition allows us to consider collections of fibers with semistable reduction.

\subsection{Torsion conditions}

As discussed in the previous section, we only need to search through configurations that are composed of collections of fibers of type $I_{v}$ in order to compute lower bounds for $\hat{h}$. Suppose that $E$ has a non-trivial torsion subgroup $E(K)_{\text {tors }}=\mathbb{Z} / 2 \mathbb{Z}$, and let $T$ be the 2-torsion point. 
The condition that $\hat{h}(T)=0$ puts a strong restriction on the possible collection of singular fibers of $E$. Recall that the components of a special fiber form a group, and we know from the list of possible groups that if there is a 2-torsion component then it is unique (see $[\mathbf{8}, \mathbf{1 0}]$ ). At each place $v$, the point $T$ meets either the identity component of the fiber $E_{v}$, or the 2-torsion component of $E_{v}$. Let $S$ and $S^{\prime}$ denote, respectively, these sets of places. We compute

$$
\hat{h}(T)=\sum_{v \in S} d_{v} B(0)+\sum_{v \in S^{\prime}} d_{v} B(1 / 2)=0 .
$$

Because $B(0)=1 / 6$ and $B(1 / 2)=-1 / 12$, it follows that

$$
2 \sum_{v \in S} d_{v}=\sum_{v \in S^{\prime}} d_{v}
$$

In the case when $G=\mathbb{Z} / 3 \mathbb{Z}$, we have $B(0)=1 / 6$ and $B(1 / 3)=B(-1 / 3)=-1 / 18$, it follows that

$$
3 \sum_{v \in S} d_{v}=\sum_{v \in S^{\prime}} d_{v}
$$

where $S$ and $S^{\prime}$ denote, respectively, the set of places at which the 3-torsion point $T$ meets identity and non-identity components of a singular fiber $E_{v}$. One can deal with any torsion group in a similar fashion, though the restriction that $d$ be small will not allow many of the 19 possibilities.

\section{Computing lower bounds for the canonical height}

In this section we translate the conditions listed in $\S \S 3.2$ and 3.4 into algorithms that search through elements of the group $\mathcal{G}$.

\subsection{Lower bounds for elliptic surfaces with 2-torsion}

We begin with elliptic surfaces with 2-torsion. Algorithm 1 describes precisely how we search through elements of $\mathcal{G}$ for configurations that might correspond to elliptic surfaces with 2 -torsion. We apply the algorithm to the cases $n=1,2,3$ and compute lower bounds.

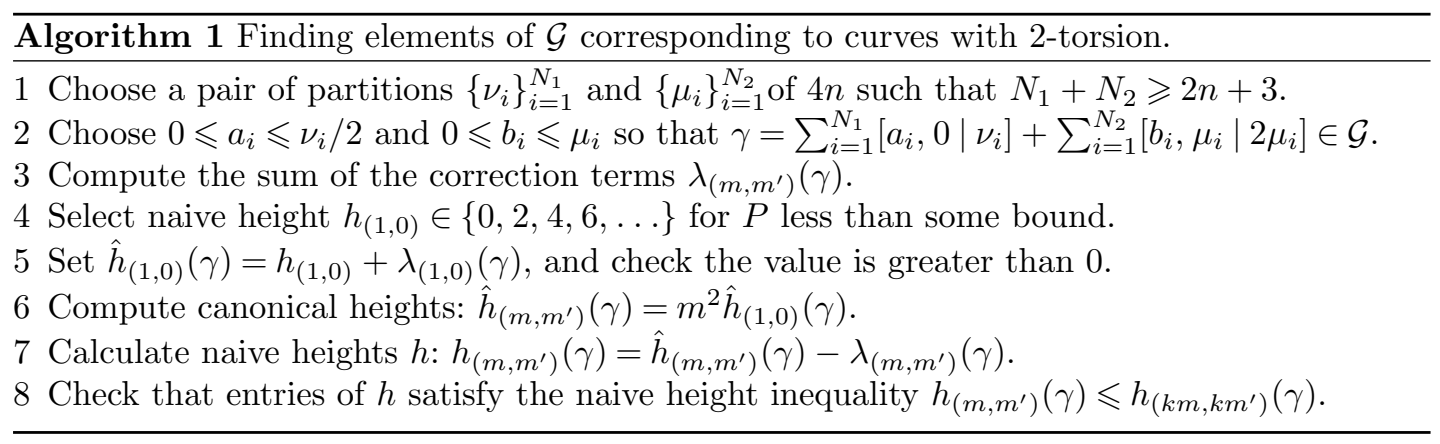

4.1.1. $n=1$. We assume that the naive height $h(P)=0$. If $h(P) \geqslant 2$ then by equation (1) we have $\hat{h}(P) \geqslant 1$, hence searching through configurations $\gamma$ with $h_{(1,0)}=0$ covers all $(E, P, T)$ with $\hat{h}(P)<1$. We find that the lower bound for $\hat{h}(P)$ occurs for

$$
\gamma=[1,2 \mid 4]+[1,1 \mid 2]+[0,1 \mid 2]+[1,0 \mid 3]+[0],
$$

with $\hat{h}_{(1,0)}(\gamma)=1 / 12$ and $\mathbf{N}(\gamma)=5$. We have $h_{(m, 0)}=0$ for $m=1, \ldots, 4$. We find no other configurations with $h_{(m, 0)}=0$ for $m=1, \ldots, 4$. The lower bound for $\hat{h}(P)$ is attained by the one parameter family of elliptic curves over $\mathbb{P}^{1}$ in Theorem 1. 
4.1.2. $n=2$. We assume that the naive height $h(P)=0$ or 2 . If $h(P) \geqslant 4$ then by equation (1) $\hat{h}(P) \geqslant 2$. Hence searching through $\gamma$ with $h_{(1,0)}=0,2$, we cover at least all $(E, P, T)$ with $\hat{h}(P)<2$. The lower bound for $\hat{h}(P)$ occurs for the configuration

$$
\gamma=[3,4 \mid 8]+[1,0 \mid 5]+[1,2 \mid 4]+[1,0 \mid 2]+2[0,1 \mid 2]+[0],
$$

with $N(\gamma)=7, h_{(m, 0)}=0$ for $m=1, \ldots, 5$ and $h_{(m, 1)}=0$ for $m=1,2,4$. Searching through our list of configurations, we find no other configuration with $h_{(m, 0)}=0$ for $m=1, \ldots, 5$. This configuration is attained by the single elliptic surface in Theorem 2 . We find one other configuration attaining the minimum:

$$
\gamma^{\prime}=[1,4 \mid 8]+[1,0 \mid 5]+[1,2 \mid 4]+[1,0 \mid 2]+2[1,1 \mid 2]+[0] .
$$

This configuration corresponds to shifting $P$ by the torsion point $T$, and in $\S 5.1$ we will see that it is equivalent to $\gamma$ under the symmetry of the moduli space in which we recover the curve.

4.1.3. $n=3$. We again assume that the naive height is $h(P)=0$ or 2 . If $h(P) \geqslant 4$ then by equation (1) we would have $\hat{h}(P) \geqslant 1$. Searching through configurations $\gamma$ with $h_{(1,0)}=0,2$, we cover at least all $(E, P, T)$ with $\hat{h}(P)<2$. The lower bound for $\hat{h}(P)$ is again $3 / 40$, and occurs for

$$
\gamma=[3,4 \mid 8]+[1,3 \mid 6]+[1,2 \mid 4]+2[0,1 \mid 2]+[1,0 \mid 5]+[1,0 \mid 3]+[1,0 \mid 2]+2[0],
$$

with $N(\gamma)=10, h_{(m, 0)}=0$ for $m=1, \ldots, 6$ and $h_{(m, 1)}=0$ for $m=1, \ldots, 4$. We find no other configurations that have $h_{(m, 0)}=0$ for $m=1, \ldots, 6$. We find one other configuration with $\hat{h}(\gamma)=3 / 40$ :

$$
\gamma^{\prime}=[1,4 \mid 8]+[2,3 \mid 6]+[1,2 \mid 4]+2[1,1 \mid 2]+[1,0 \mid 5]+[1,0 \mid 3]+[1,0 \mid 2]+2[0] .
$$

This configuration, however, is equivalent to $\gamma$. In $\S 5.1$ we will see that this configuration corresponds to shifting $P$ to $P+T$ on the curve in Theorem 3.

\subsection{Lower bounds for elliptic surfaces with 3-torsion}

Algorithm 2 describes our search through elements of $\mathcal{G}$ for configurations that could correspond to elliptic surfaces with 3 -torsion. We apply the algorithm to the cases $n=1,2$ and compute lower bounds.



4.2.1. $n=1$. We again assume that the naive height $h(P)=0$. As in $\S 4.1$, searching through configurations with $h_{(0,1)}=0$ covers all $(E, P, T)$ with $\hat{h}(P)<1$. We generate a list of configurations. A lower bound for $\hat{h}(P)$ occurs for

$$
\gamma=[1,2 \mid 3]+[1,1 \mid 3]+[0,2 \mid 3]+[1,0 \mid 2]+[0] .
$$


This configuration has $\hat{h}_{(1,0)}(\gamma)=1 / 6$, with $h_{\left(m, m^{\prime}\right)}(\gamma)=0$ for

$$
\left(m, m^{\prime}\right)=(1,0),(2,0),(3,0),(1,1),(2,1),(1,2),(2,2) .
$$

We search through our list and see that no other configuration has $h_{(m, 0)}=0$ for $m=1,2,3$. The configuration $\gamma$ is realized by the one parameter family of rational elliptic surfaces in Theorem 4.

4.2.2. $\quad n=2$. As in $\S 4.1 .2$, we assume that the naive height $h(P)$ is 0 or 2 , and generate a list of configurations. We find that a lower bound for the canonical height occurs for the configuration

$$
\gamma=[3,4 \mid 6]+[1,2 \mid 6]+[1,4 \mid 6]+[1,0 \mid 3]+3[0] .
$$

The configuration has $\hat{h}_{(1,0)}(\gamma)=1 / 6$, with $h_{\left(m, m^{\prime}\right)}=0$ for

$$
\left(m, m^{\prime}\right)=(1,0),(2,0),(1,1),(1,2),(2,1),(2,2),(3,1),(3,2) .
$$

In addition, no other configuration in our list has $h_{\left(m, m^{\prime}\right)}=0$ for the same $\left(m, m^{\prime}\right)$. The configuration is realized by the one parameter family of $K 3$ elliptic surfaces in Theorem 5 .

\section{Explicit modular parametrizations}

In this section we derive the explicit equations in Theorems $1-5$. We begin by constructing projective models.

\subsection{Elliptic curves with 2-torsion}

We find explicit equations for the optimal 2-torsion surfaces for $n=1,2,3$, using the fact that they have a section $P$ with the first several $m P$ and $m P+T$ integral. We first construct a suitable moduli space in which to work, and then recover the surfaces as curves in this moduli space. We parameterize triples $(E, P, T)$ of elliptic curves with a 2 -torsion point $T$ and a point $P$ with the property that $P, 2 P, P+T$, and $2 P+T$ are all integral by an open subset in $\mathbb{P}^{2}$. The $n=1,2,3$ surfaces we want to find will then be curves of degree $n=1,2,3$ in this moduli space.

We begin with a curve $E$ with a rational point $T=(0,0)$ placed at the origin:

$$
y^{2}+a_{1} x y+a_{3} y=x^{3}+a_{2} x^{2}+a_{4} x .
$$

The point $T$ is a 2-torsion point precisely when the slope of the tangent to $E$ at $T$ is vertical. The slope of this line is $a_{4} / a_{3}$, and this line is vertical when $a_{3}=0$. Next, replacing $y$ by $y-a_{1} x / 2$ eliminates the $a_{1}$ term. Our curve is now of the form

$$
y^{2}=x^{3}+a_{2} x^{2}+a_{4} x .
$$

Let $P=(X, Y)$ be another integral point of $E$ that is non-torsion. The point $P+T$ is integral only if the slope of the secant line through $P$ and $T$ has integral slope. This slope is $Y / X$, and we write $Y=a X$. Writing the Weierstrass equation that the coordinates of $T$ must satisfy, we obtain

$$
X\left(X^{2}+\left(a_{2}-a^{2}\right) X+a_{4}\right)=0 .
$$

Since $X$ cannot be 0 , we find that $a_{4}=X\left(a^{2}-a_{2}-X\right)$.

Next we impose the condition that $2 P$ is an integral point. This occurs when the tangent to $E$ at $P$ has integral slope. This line has slope

$$
\frac{2 x^{2}+\left(a_{2}+a^{2}\right) x}{2 y}=\frac{2 x+\left(a_{2}+a^{2}\right)}{2 a} .
$$

Thus $a$ divides $2 X+a_{2}$ and we my write $2 X+a_{2}=a(a+c)$ or $a_{2}=a(a+c)-2 X$ for some $c$. 


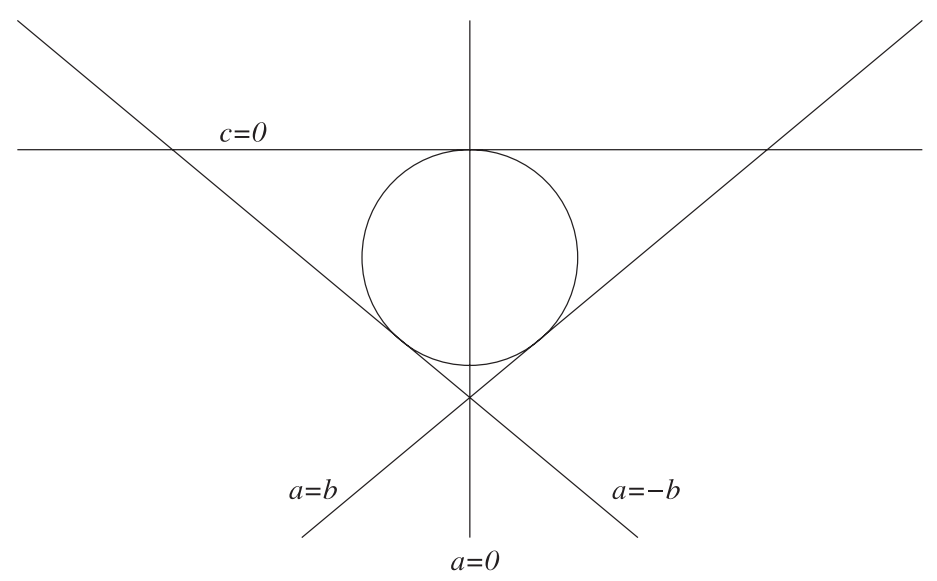

FIGURE 1. $\Delta$ vanishes on the conic $4 c(b-c)=a^{2}$ with multiplicity 1 , on $a=0$ (vertical axis) and $b= \pm a$ (diagonals) with multiplicity 2 , and on $c=0$ (line at infinity) with multiplicity 4 . The conic meets the lines $a=0, c=0$ at $(0: 1: 0)$; the involution $a \leftrightarrow a$ takes $P=((a+b) c,(a+b) a c)$ to $P+T$.

Finally we impose the condition that $2 P+T$ is integral, or the slope of the line through $2 P$ and $T$ is integral. This slope is

$$
\frac{2}{c} X+\left(-a-\frac{1}{2} c\right)
$$

and it follows that $c$ divides $X$. We write $X=c(a+b)$ for some $b$, which gives us

$$
\begin{aligned}
& y^{2}=x^{3}+\left(a^{2}-2 b c\right) x^{2}+\left(b^{2}-a^{2}\right) c^{2} x \\
& P=((a+b) c,(a+b) a c), \quad T=(0,0),
\end{aligned}
$$

with $\Delta=16 a^{2}(b-a)^{2}(b+a)^{2} c^{4}\left(a^{2}-4 c(b-c)\right)$. This parametrizes triples $(E, P, T)$ of elliptic curves with a 2 -torsion point $T$ and a rational point $P$ with the property that $P, 2 P, P+T$, and $2 P+T$ are all integral: changing $(a, b, c)$ to $(\lambda a, \lambda b, \lambda c)$ yields an isomorphic curve and we have a parametrization of the $(E, P, T)$ moduli space by an open subset in $\mathbb{P}^{2}$. A curve $C$ of degree $n$ in this $\mathbb{P}^{2}$ amounts to an elliptic surface $\mathcal{E} \longrightarrow C$ of discriminant degree $12 n$, with sections $P$ and $T$ such that $P, 2 P, P+T$, and $2 P+T$ are integral and $2 T=0$. The surface will have a configuration of fibers and sections $P, T$ given by

$$
\gamma=n[0,1 \mid 2]+n[1,1 \mid 2]+n[1,0 \mid 2]+n[1,2 \mid 4]+2 n[0],
$$

corresponding to the $n$ points at which $C$ meets the lines $a= \pm b, a=0$ and $c=0$, and the $2 n$ points at which $C$ meets the conic $4 c(b-c)=a^{2}$. We recover the optimal surfaces in the 2-torsion case as curves in this $\mathbb{P}^{2}$ model. The involution of $\mathbb{P}^{2}$ that corresponds to interchanging $P \leftrightarrow P+T$ is given by $(a: b: c) \longrightarrow(-a: b: c)$. The optimal $n=1,2,3$ surfaces sit as curves of degree $n$ in this $\mathbb{P}^{2}$. See Figure 1.

5.1.1. $n=1$. The optimal $n=1$ configuration is

$$
\gamma=[1,2 \mid 4]+[1,1 \mid 2]+[0,1 \mid 2]+[1,0 \mid 3]+[0],
$$

with $\hat{h}(P)=1 / 12$. This configuration has conductor $\mathbf{N}(\gamma)=5$ and hence should be attained by a one parameter family of rational elliptic surfaces. Suppose that

$$
\begin{aligned}
& \lambda: \mathbb{P}^{1} \longrightarrow \mathbb{P}^{2} \\
& \left(s: s^{\prime}\right) \mapsto(A: B: C)
\end{aligned}
$$


denotes a line in $\mathbb{P}^{2}$ that gives rise to such a rational elliptic surface. Each $A, B, C$ is a homogenous linear form in $s, s^{\prime}$. Without loss of generality we may assume that $A=s$ and $B=s^{\prime}$. We see that the only way to obtain a fiber of type $[1,0 \mid 3]$ is to force $\lambda$ through the point $(0: 1: 1)$, where the line $a=0$ and the conic $4 c(b-c)=a^{2}$ meet. Thus we may set $C=q s+s^{\prime}$. This gives us a one parameter family of lines

$$
\begin{aligned}
\lambda(q) & : \mathbb{P}^{1} \longrightarrow \mathbb{P}^{2} \\
\quad\left(s: s^{\prime}\right) & \mapsto\left(s: s^{\prime}: q s+s^{\prime}\right),
\end{aligned}
$$

where each line in this family gives rise to a rational elliptic surface $E$ with a 2-torsion section $T$ and a section $P$ of height $\hat{h}(P)=1 / 12$. Setting $t=s / s^{\prime}$ gives the equations in Theorem 1 .

5.1.2. $n=2$. The optimal $n=2$ configuration is given by

$$
\gamma=[3,4 \mid 8]+[1,0 \mid 5]+[1,2 \mid 4]+[1,0 \mid 2]+2[0,1 \mid 2]+[0],
$$

with $\hat{h}(P)=3 / 40$. This configuration has conductor $\mathbf{N}(\gamma)=7$ and hence should be given by a single $K 3$ elliptic surface. Suppose that

$$
\begin{aligned}
& \lambda: \mathbb{P}^{1} \longrightarrow \mathbb{P}^{2} \\
& \left(s: s^{\prime}\right) \mapsto(A: B: C)
\end{aligned}
$$

denote a conic in $\mathbb{P}^{2}$ that gives rise to such a $K 3$ elliptic surface. Each $A, B, C$ is homogeneous form of degree two in $s, s^{\prime}$.

A generic curve of degree two in this moduli space will give rise to a surface having configuration $\gamma$ of fibers and sections $P, T$ given by

$$
2[0,1 \mid 2]+2[1,1 \mid 2]+2[1,0 \mid 2]+2[1,2 \mid 4]+4[0] .
$$

We will arrive at the optimal configuration by forcing the curve $\lambda$ to go through some specific points in the $\mathbb{P}^{2}$ model.

The only way to obtain a fiber of type $[3,4 \mid 8]$ is to merge a fiber of type $[1,2 \mid 4]$ with two fibers of type $[1,1 \mid 2]$. This forces $\lambda$ to go through the point $(1:-1: 0)$, where the lines $c=0$ and $a=-b$ intersect, meeting the line $a=-b$ with multiplicity 2 . Thus we write

$$
\begin{aligned}
\lambda(q) & : \mathbb{P}^{1} \longrightarrow \mathbb{P}^{2} \\
\quad\left(s: s^{\prime}\right) & \mapsto\left(A, s^{2}-A, s s^{\prime}\right) .
\end{aligned}
$$

This brings us down to a three parameter family whose generic member has a section $P$ of height $3 / 8$. We use the remaining free parameters to get to the desired configuration, and bring the height of $P$ down to $3 / 40$.

The only way we can obtain a fiber $[1,0 \mid 5]$ is to merge a fiber of type $[1,0 \mid 2]$ with three fibers of type [0]. This forces $\lambda$ to meet the conic $4 c(b-c)=a^{2}$ triply at its point of intersection with the line $a=0$, which implies that

$$
-A^{2}-4 s s^{\prime} A+\left(4 s^{\prime} s^{3}-4 s^{2} s^{2}\right)
$$

has a common factor with $A$. Then $\left(s-s^{\prime}\right) s^{2} s^{\prime}$ has a factor in common with $A$, which forces $\left(s-s^{\prime}\right) \mid A$, and we may write $A=r\left(s-s^{\prime}\right)$. We force the above equation to have two more factors of $\left(s-s^{\prime}\right)$. Dividing through by $\left(s-s^{\prime}\right)$ we are left with

$$
4 s^{\prime} s^{2}+\left(-4 r s^{\prime}-r^{2}\right) s+r^{2} s^{\prime}=-\left(s-s^{\prime}\right) r^{2}+4(s-r) s s^{\prime} .
$$

The linear forms $s$ and $s^{\prime}$ cannot divide each other, as $\gamma$ would have a fiber of type $I_{12}$ in that situation. This implies that $\left(s-s^{\prime}\right) \mid(s-r)$, and we write $r=q s+(1-q) s^{\prime}$. Substituting this in for $r$ and again dividing through by $s-s^{\prime}$, we obtain

$$
-q^{2} s^{2}+\left(2 q^{2}-6 q+4\right) s^{\prime} s+\left(-q^{2}+2 q-1\right) s^{\prime 2}=\left(s-s^{\prime}\right)\left(s^{\prime}(1-q)^{2}-q^{2} s\right)+s s^{\prime}(3-4 q) .
$$


This forces $q=3 / 4$, and gives us the desired conic in our moduli space:

$$
\begin{aligned}
& \lambda: \mathbb{P}^{1} \longrightarrow \mathbb{P}^{2} \\
& \left(s: s^{\prime}\right) \mapsto\left(\left(s-s^{\prime}\right)\left(s^{\prime}+3 s\right):\left(s+s^{\prime}\right)^{2}: 4 s s^{\prime}\right) .
\end{aligned}
$$

This conic gives rise to the $K 3$ elliptic surface in Theorem 2 by setting $t=s / s^{\prime}$.

5.1.3. $n=3$. The optimal $n=3$ configuration is given by

$$
\gamma=[3,4 \mid 8]+[1,3 \mid 6]+[1,2 \mid 4]+2[0,1 \mid 2]+[1,0 \mid 5]+[1,0 \mid 3]+[1,0 \mid 2]+2[0],
$$

with $\hat{h}(P)=3 / 40$. The conductor $\mathbf{N}(\gamma)=11$, and hence $\gamma$ should be given by a one parameter family of elliptic surfaces. Let

$$
\begin{aligned}
& \lambda: \mathbb{P}^{1} \longrightarrow \mathbb{P}^{2} \\
& \left(s: s^{\prime}\right) \mapsto(A: B: C)
\end{aligned}
$$

denote a rational cubic in $\mathbb{P}^{2}$ that gives rise to such an elliptic surface. Each $A, B, C$ is a homogeneous cubic form in $s, s^{\prime}$.

A generic rational cubic $C$ will give rise to an elliptic surface with configuration $\gamma$ of fibers and sections $P, T$ given by

$$
\gamma=3[0,1 \mid 2]+3[1,1 \mid 2]+3[1,0 \mid 2]+3[1,2 \mid 4]+6[0],
$$

corresponding to the three points at which $C$ meets the lines $a= \pm b, a=0$ and $c=0$, and the six points at which $C$ meets the conic $4 c(b-c)=a^{2}$. We again arrive at the optimal $n=3$ configuration by forcing the curve $\lambda$ to go through some specific points.

For the surface corresponding to $\lambda$ to have a fiber of type $[3,4 \mid 8]$, we must merge a fiber of type $[1,2 \mid 4]$ on the line $c=0$ with two fibers of type $[1,1 \mid 2]$ on the line $a=-b$. This forces $\lambda$ through the point $(1:-1: 0)$, meeting the line $a=-b$ with multiplicity two at that point. Hence we can write $B=s^{2} s^{\prime}-A$ and $C=s c_{2}$, where $c_{2}$ is a homogeneous quadratic form in $s, s^{\prime}$. This ensures a fiber of type $[3,4 \mid 8]$ at $s=0$.

Next, to obtain fibers of type $[1,0 \mid 3]$ and $[1,0 \mid 5]$, we must merge fibers of type $[1,0 \mid 2]$ on the line $a=0$ with fibers of type [0]. This forces $\lambda$ to meet the conic $4 c(b-c)=a^{2}$ at the points $(0: 0: 1)$ and $(0: 1: 1)$, meeting the conic with multiplicity three at $(0: 1: 1)$. We force $A^{2}-4 C(B-C)$ to have two linear factors in common with $A$. We calculate

$$
4 C(B-C)=4 A c_{2} s-4 s^{2} c_{2}\left(c_{2}-s s^{\prime}\right) .
$$

This forces $A$ to have two factors in common with $s^{2} c_{2}\left(c_{2}-s s^{\prime}\right)$. This forces $\left(c_{2}-s s^{\prime}\right) \mid A$ and we write $A=r\left(s s^{\prime}-c_{2}\right)$. This promotes two of the three $[1,0 \mid 2]$ fibers at $A=0$ to $[1,0 \mid 3]$ fibers.

To obtain a fiber of type $[1,3 \mid 6]$, we must merge another fiber of type $[1,2 \mid 4]$ on the line $c=0$ with a fiber of type $[0,1 \mid 2]$ on the line $a=b$. This forces $A-B$ to have a factor in common with $C=s c_{2}$. Since

$$
A-B=s s^{\prime}(2 r-s)-2 t c_{2},
$$

and we already have the desired fiber type at $s=0$, we see that $(s+2 r) \mid c_{2}$. We set $c_{2}=$ $u(2 r-s)$. This gives a fiber of type $[1,0 \mid 6]$ at $s=2 r$, and forces $\lambda$ through the point $(1: 1: 0)$.

Next we try to promote one of the $[1,0 \mid 3]$ fibers to a $[1,0 \mid 5]$. This means that one of the two fibers at $A / r=s s^{\prime}-c_{2}=s s^{\prime}-u(2 r-s)=0$ must meet the conic $a^{2}-4 c(b-c)$ to order three. This forces $A / r$ to have a factor in common with $C(B-C) /(A / r)$. We write

$$
C(B-C) /(A / r)=s u(s-r)(2 r-s) .
$$

We already have the desired fiber types at $s=0, u=0$, and $2 r=s$, so the only possible way to force a factor of $s s^{\prime}-u(2 r-s) r$ to share a factor with the quotient above is to force $s-r$ to 
divide $s s^{\prime}-u(2 r-s) r$. This implies that $s-r \mid r\left(s^{\prime}-u\right)$. Since at this point we already have the desired fiber type at $r=0$ as well, it must be the case that $s-r \mid s^{\prime}-u$. We set $r=q^{\prime}\left(s^{\prime}-u\right)+s$. Replacing $s^{\prime}$ by $s^{\prime} / q^{\prime}$, and $u$ by $u^{\prime} / q^{\prime}$, and then changing the Weierstrass equation by $1 / q^{\prime}$, we can eliminate $q^{\prime}$ from our equations.

We again compute $A^{2}-4 C(B-C)$ and divide through by the factors $(2 u-s)$ and $\left(s^{\prime}-u\right)^{2}$ to obtain

$$
-s^{3}-2 s^{\prime} s^{2}+\left(-s^{\prime 2}-2 u s^{\prime}+3 u^{2}\right) s+\left(2 u s^{\prime 2}-4 u^{2} s^{\prime}+2 u^{3}\right) .
$$

We force the cubic above to have one more factor of $\left(s^{\prime}-u\right)$ in order to obtain the desired $[1,0 \mid 5]$ fiber at $s^{\prime}=u$. This forces $s^{\prime}-u$ to divide $s^{2}(s-2 u)$ and this $s^{\prime}-u \mid s-2 u$. We set $u=q\left(s+2 s^{\prime}\right)+s^{\prime}$ to obtain our curve

$$
\begin{aligned}
& \lambda: \mathbb{P}^{1} \longrightarrow \mathbb{P}^{2} \\
& \left(s: s^{\prime}\right) \mapsto(A: B: C) \\
& A=q\left(2 s^{\prime}+s\right)\left((1-q) s-2 q s^{\prime}\right)\left((4 q+2) s^{\prime}+(2 q-1) s\right) \\
& B=s^{2} s^{\prime}-q\left(2 s^{\prime}+s\right)\left((1-q) s-2 q s^{\prime}\right)\left((4 q+2) s^{\prime}+(2 q-1) s\right) \\
& C=s\left(2 q s^{\prime}+s^{\prime}+q s\right)\left((1-2 q) s-4 q s^{\prime}\right) .
\end{aligned}
$$

This cubic gives rise to the elliptic surface in Theorem 3 by setting $t=s / s^{\prime}$.

\subsection{Elliptic curves with 3-torsion}

We find equations for the general elliptic curve $E / \mathbb{Q}$ with a 3 -torsion point $T$ and another point $P$ such that $P+n T$ is integral for each $n=0,1,2$. We parametrize this set of $(E, P, T)$ by an open in $\mathbb{P}^{2}$. We then recover the $n=1,2$ surfaces as curves in this moduli space.

As in $\S 5.1$, one can easily find equations for the general elliptic curve with a 3 -torsion point $T=(0,0)$ placed at the origin:

$$
y^{2}+a_{1} x y+a_{3} y=x^{3} .
$$

Suppose the point $P$ has coordinates $(X, Y)$. Then since $P+T$ is integral, the slope of the line through $P$ and $T$ must be integral. Hence $X \mid Y$ and we write $Y=-a X$ for some constant $a$. Next, since $2 T=\left(0, a_{3}\right)$ and $P+2 T$ is also integral, we know that the slope of the line through $P$ and $2 T$ is integral. This slope is equal to $-a+x / a_{3}$, and we write $a_{3}=-c X$. Finally, writing the Weierstrass equation that the coordinates of $P$ satisfy, we find that

$$
Y^{2}+a_{1} X Y+a_{3} X-X^{3}=X^{2}\left(a^{2}-a_{1} a-a c-X\right)=0
$$

and we find that $X=a^{2}-a_{1} a+a c$. Finally we set $a_{1}=a+b+c$.

The conditions on $P$ then amount to $a_{1}=a+b+c, a_{3}=a b c$, with $P$ at $\left(-a b, a^{2} b\right)$. This gives us our model

$$
\begin{gathered}
E: y^{2}+(a+b+c) x y+a b c y=x^{3}, \\
P=\left(-a b, a^{2} b\right) .
\end{gathered}
$$

The translates of $P$ by multiples of $T$ are given by cyclically permuting $\{a, b, c\}$, and their negatives by the odd permutations. The lines $a=0, b=0, c=0$ give $[1,2 \mid 3],[1,1 \mid 3]$, and $[0,2 \mid 3]$ fibers, and the residual factor of the discriminant is the singular cubic $(a+b+c)^{3}=$ $27 a b c$, meeting $a=0, b=0$ and $c=0$ to order three.

A generic curve $C$ of degree $n$ in this moduli space corresponds to an elliptic surface $\mathcal{E} \longrightarrow C$ of discriminant degree $12 n$ with sections $P$, and $T$ such that $P, P+T$, and $P+2 T$ are integral and $3 T=0$. The surfaces have a configuration of fibers and sections given by

$$
\gamma=[1,2 \mid 3]+[1,1, \mid 3]+[0,2 \mid 3]+3[0],
$$




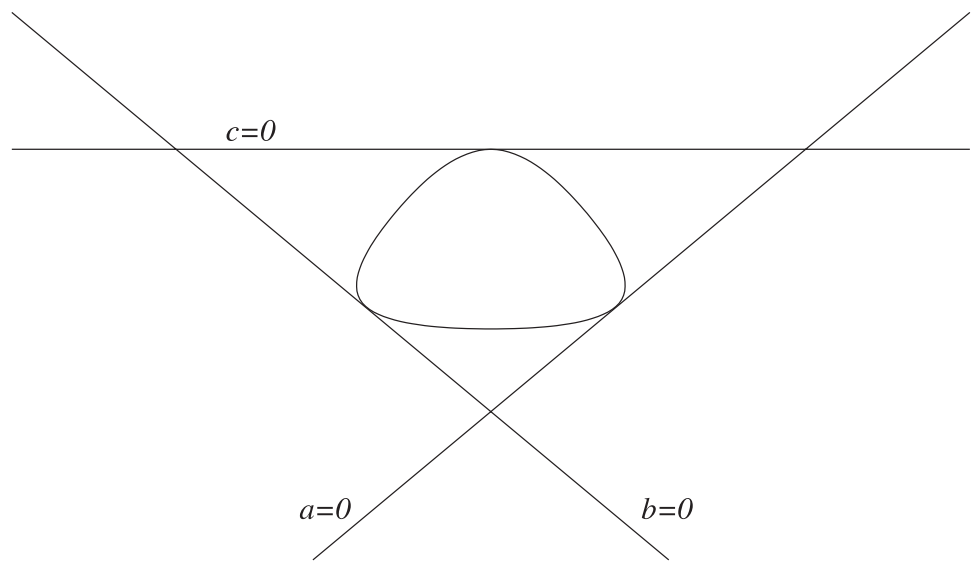

Figure 2. $\Delta$ vanishes on the cubic $(a+b+c)^{3}=27 a b c$ with multiplicity 1 , on $a=0$ and $b=0$ (diagonals) with multiplicity 3 , and on $c=0$ (line at infinity) with multiplicity 3 . The cubic meets the line $a=0$ at $(0:-1: 1), b=0$ at $(-1: 0: 1)$ and $c=0$ at $(0:-1: 1)$. Cyclically permuting $\{a, b, c\}$ to $\{c, a, b\}$ and $\{b, c, a\}$ sends $P=\left(-a b, a^{2} b\right)$ to $P+T$ and $P+2 T$.

corresponding to the points at which the curve meets $a=0, b=0, c=0$, and the cubic $(a+b+c)^{3}=27 a b c$. See Figure 2 .

5.2.1. $n=1$. The optimal $n=1$ configuration is given by

$$
[1,2 \mid 3]+[1,1 \mid 3]+[0,2 \mid 3]+[1,0 \mid 2]+[0],
$$

with $\hat{h}(P)=1 / 6$. The conductor $\mathbf{N}(\gamma)=5$, and hence $\gamma$ should be realized by a one parameter family of elliptic surfaces. Let

$$
\begin{aligned}
& \lambda: \mathbb{P}^{1} \longrightarrow \mathbb{P}^{2} \\
& \left(s, s^{\prime}\right) \mapsto(A: B: C)
\end{aligned}
$$

denote a line in $\mathbb{P}^{2}$ that gives rise to such an elliptic surface. Each $A, B, C$ is of homogeneous linear form in $s, s^{\prime}$.

A generic line in $\mathbb{P}^{2}$ will correspond to a rational elliptic surface with configuration $\gamma$ of fibers and sections $P, T$ given by

$$
[1,2 \mid 3]+[1,1 \mid 3]+[0,2 \mid 3]+3[0],
$$

corresponding to the points at which the line meets $a=0, b=0, c=0$, and the cubic $(a+b+c)^{3}=27 a b c$. The cubic intersects itself at $(1: 1: 1)$, and forcing the line through this point merges two [0] fibers, giving the desired configuration. This gives the one parameter family of lines in $\mathbb{P}^{2}$ through $(1: 1: 1)$

$$
\begin{aligned}
& \lambda: \mathbb{P}^{1} \longrightarrow \mathbb{P}^{2} \\
& \left(s: s^{\prime}\right) \mapsto\left(s: s^{\prime}: q s+(1-q) s^{\prime}\right),
\end{aligned}
$$

and this family of lines gives rise to the family of rational elliptic surfaces in Theorem 4 (again we set $t=s / s^{\prime}$ in the statement of the theorem).

5.2.2. $n=2$. The optimal $n=2$ configuration is given by

$$
[3,4 \mid 6]+[1,2 \mid 6]+[1,4 \mid 6]+[1,0 \mid 3]+3[0]
$$


with $\hat{h}(P)=1 / 6$ and conductor $\mathbf{N}(\gamma)=7$. We search for a zero parameter family of $K 3$ elliptic surfaces with the above fiber configuration. Let

$$
\begin{aligned}
& \lambda: \mathbb{P}^{1} \longrightarrow \mathbb{P}^{2} \\
& \left(s: s^{\prime}\right) \mapsto(A: B: C)
\end{aligned}
$$

denote a conic in $\mathbb{P}^{2}$ that gives rise to such an elliptic surface. Each $A, B, C$ is of homogeneous linear form in $s, s^{\prime}$.

Generically, the curve $\lambda$ gives rise to an $K 3$ elliptic surface with fiber configuration

$$
2[1,2 \mid 3]+2[1,1 \mid 3]+2[0,2 \mid 3]+6[0] .
$$

We again force $\lambda$ to go through some specific points in our $\mathbb{P}^{2}$ model to arrive at the desired one parameter family of $K 3$ elliptic surfaces. At the points at which $\lambda$ meets the lines $a=0$, $b=0$ and $c=0$, the torsion point $T$ will meet a non-identity component of an $I_{3}$ fiber. One can see that the only way to force the desired $I_{6}$ fibers is to force $\lambda$ to go through the three intersection points of these three lines. This amounts to setting $A=s r, B=r u$ and $C=s u$, bringing our configuration down to

$$
[3,4 \mid 6]+[1,4 \mid 6]+[1,2 \mid 6]+6[0] .
$$

We know that the point $P$ meets the component $v\left(r^{3} s u(s-u) / 2\right)$ of the fiber at $v$. This tells us that the fiber of type $[1,0 \mid 3]$ must occur at $s=u$, since $P$ meets a non-identity component of that fiber.

We force

$$
\left(r^{3}+3 u r^{2}+3 u^{2} r+u^{3}\right) s^{3}+\left(3 u r^{3}-21 u^{2} r^{2}+3 u^{3} r\right) s^{2}+\left(3 u^{2} r^{3}+3 u^{3} r^{2}\right) s+u^{3} r^{3} .
$$

to have three factors in common with $s-u$. We compute the remainder upon dividing the above expression by $s-u$, and we obtain

$$
u^{3}(u-r)^{2}(u+8 r)
$$

Hence we know that $(s-u) \mid(u-r)$. We set $r=q(s-u)+u$. This promotes the fiber at $s=u$ to one of type $[1,0 \mid 2]$. Finally, we divide $(A+B+C)^{3}-27 A B C$ by $(s-u)^{2}$, obtaining

$$
\begin{aligned}
& q^{3} s^{4}+\left(2 q^{3}+6 q^{2}\right) u s^{3}+\left(-12 q^{2}+12 q\right) u^{2} s^{2} \\
& \quad+\left(-2 q^{3}+12 q^{2}-18 q+8\right) u^{3} s+\left(-q^{3}+3 q^{2}-3 q+1\right) u^{4} .
\end{aligned}
$$

Computing the remainder upon division of the above expression by $(s-u)$, we obtain

$$
9\left(q^{2}-q+1\right) u^{4}
$$

TABLE 1. 3-torsion configurations with small $\mathbf{N}$.

\begin{tabular}{cc}
\hline Configuration & $\hat{h}(\gamma)$ \\
\hline$[3,2 \mid 6]+[1,2 \mid 6]+[1,2 \mid 3]+[1,0 \mid 6]$ & $1 / 6$ \\
{$[3,2 \mid 6]+[1,2 \mid 6]+[1,4 \mid 6]+[1,0 \mid 4]+2[0]$} & $1 / 12$ \\
{$[1,3 \mid 9]+[3,2 \mid 6]+[1,2 \mid 3]+[1,0 \mid 5]+[0]$} & $13 / 90$ \\
{$[1,3 \mid 9]+[3,2 \mid 6]+[1,2 \mid 3]+[1,0 \mid 6]$} & $1 / 9$ \\
{$[4,3 \mid 9]+[1,6 \mid 9]+[1,0 \mid 4]+2[0]$} & $5 / 36$ \\
{$[4,3 \mid 9]+[1,6 \mid 9]+[1,0 \mid 5]+[0]$} & $4 / 45$ \\
{$[2,3 \mid 9]+[2,6 \mid 9]+[1,0 \mid 6]$} & $1 / 18$ \\
\hline
\end{tabular}


The two roots of the polynomial $q^{2}+q+1$ give us the pair of $K 3$ elliptic surfaces in Theorem 5 :

$$
\begin{aligned}
& \lambda: \mathbb{P}^{1} \longrightarrow \mathbb{P}^{2} \\
& \left(s: s^{\prime}\right) \mapsto\left(q s^{2}+(-q+1) s^{\prime} s: q s^{\prime} s+(-q+1) s^{\prime 2}: s^{\prime} s\right) .
\end{aligned}
$$

This completes the proof of Theorem 5 . Setting $t=s / s^{\prime}$ yields the equations in the statement of the theorem.

\subsection{Surfaces over higher genus curves}

In this section we prove that for $d=12$ and $d=24$, the minimum heights over elliptic surfaces over $\mathbb{P}^{1}$ are the minimum for elliptic surfaces over curves of any genus. The fibers and sections of an elliptic surface $E$ over a curve of genus $g>0$ are subject to all of the combinatorial constraints laid out in $\S \S 3.2$ and 3.4 except Shioda's inequality in $\S 3.2$ which says that the conductor degree $N$ of $E$ is bounded below by

$$
N \geqslant(d / 6)+\chi(C)+1 .
$$

In the case where $g \geqslant 1$, the Euler characteristic $\chi(C)=2-2 g$ can be negative, and Shioda's inequality becomes trivial. None of the other conditions, however, depend on the genus of the base curve.

Hence Algorithms 1 and 2 change only in step 1, where we assume that $\mathbf{N}(\gamma) \geqslant 2 n+3=7$. We remove this restriction, and generate a list of configurations that could possibly correspond to elliptic surfaces curves of higher genus.

In the case that $E(K)_{\text {tors }}=\mathbb{Z} / 2 \mathbb{Z}$ and $d=12$ or $d=24$, we find no new configurations. Hence we automatically get the result over higher-genus curves. In the case where $E(K)_{\text {tors }}=$ $\mathbb{Z} / 3 \mathbb{Z}$ and $d=12$ we find no new configurations. However for $d=24$, we find several new configurations. Each of the configurations has $h_{(1,0)}=h_{(1,1)}=h_{(1,2)}=0$, and we list them in Table 1 . Hence if any of these configurations is realized by an elliptic surface of discriminant degree 24 , this elliptic surface will correspond to a curve of degree two in our $\mathbb{P}^{2}$ model. A curve of degree two in $\mathbb{P}^{2}$ is rational, and hence these surfaces will in fact be $K 3$. This contradicts part (i) of Theorem 5.

Acknowledgements. Many thanks go to Noam Elkies for numerous discussions. Thanks also go to David Harvey and Matthias Schuett for helpful comments, Michael Burr for help with the illustrations and the anonymous referee for many comments which improved the paper.

\section{References}

1. D. A. Cox and S. Zucker, 'Intersection numbers of sections of elliptic surfaces', Invent. Math. 53 (1979) no. 1, 1-44; MR 538682(81i:14023.

2. N. D. ElKIES, 'Points of low canonical height on elliptic curves and surfaces', 2001, http://math.harvard.edu/ elkies/loht.pdf.

3. N. D. ElkiEs, 'Points of low height on elliptic curves and surfaces. I. Elliptic surfaces over $\mathbb{P}^{1}$ with small d', Algorithmic number theory, Lecture Notes in Computer Science 4076 (Springer, Berlin, 2006) 287-301; MR 2282931(2008e:11082).

4. N. D. ElkiES, 'Points on elliptic curves with several integral multiples: algebra, geometry and some applications', 2006, http://math.harvard.edu/ elkies/ants7_loht.pdf.

5. M. Hindry and J. H. Silverman, 'The canonical height and integral points on elliptic curves', Invent. Math. 93 (1988) no. 2, 419-450; MR 948108(89k:11044).

6. S. JAIN, 'The minimum canonical height on an elliptic curve over $\mathbb{C}(t)$ ', http://cims.nyu.edu/ jain/record.pdf.

7. S. JAIN, 'Minimal regulators for rank-2 subgroups of rational and $K 3$ elliptic surfaces', Experiment. Math. 18 (2009) no. 4, 429-447; MR 2583543.

8. K. Kodaira, 'On compact analytic surfaces. II, III', Ann. of Math. (2) 77 (1963) 563-626; K. KodAIRA, 'On compact analytic surfaces. II, III', Ann. of Math. (2) 78 (1963), 1-40; MR 0184257(32\#1730).

9. S. Lang, Elliptic curves: Diophantine analysis, Grundlehren der Mathematischen Wissenschaften [Fundamental Principles of Mathematical Sciences] 231 (Springer, Berlin, 1978); MR 518817(81b:10009). 
10. A. Néron, 'Modèles minimaux des variétés abéliennes sur les corps locaux et globaux', Publ. Math. Inst. Hautes Études Sci. 21 (1964) 128; MR 0179172(31\#3423).

11. K.-I. NishiYama, 'The minimal height of Jacobian fibrations on K3 surfaces', Tohoku Math. J. (2) 48 (1996) no. 4, 501-517; MR 1419081(97k:14037).

12. K. Oguiso and T. Shioda, 'The Mordell-Weil lattice of a rational elliptic surface', Comment. Math. Univ. St. Pauli. 40 (1991) no. 1, 83-99; MR 1104782(92g:14036).

13. J. H. Silverman, Advanced topics in the arithmetic of elliptic curves, Graduate Texts in Mathematics, vol. 151 (Springer, New York, 1994); MR 1312368(96b:11074).

14. J. T. TATE, 'The arithmetic of elliptic curves', Invent. Math. 23 (1974) 179-206; MR 0419359(54\#7380).

\section{Sonal Jain}

Courant Institute of Mathematical Sciences

251 Mercer Street, New York, NY 10012

USA

jain@courant.nyu.edu 